\title{
Proteção medular em cirurgia da aorta descendente com uso de "bio-pump" e exsangüinação controlada
}

Henrique MURAD ${ }^{\star * *}$, José L. FEITOSA ${ }^{*}$, Rubens GIAMBRONIFILHO ${ }^{\star \star *}$, José Augusto de AZEVEDO ${ }^{\star * *}$,

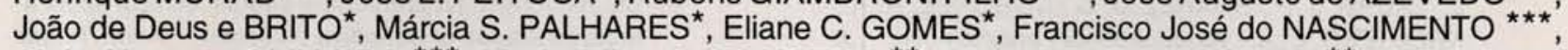
Diniz Alves de OLIVEIRA ${ }^{\star \star \star}$, Maria Elisa G. FONSECA ${ }^{\star \star}$, Amauri Dias do COUTO ${ }^{\star \star}$, Mauro Paes Leme de $S A{ }^{*}$, Juscileide M. de Araújo PIRES*.

RBCCV 44205-237

MURAD, H.; FEITOSA, J. L.; GIAMBRONI FILHO, R.; AZEVEDO, J. A.; BRITO, J. D.; PALHARES, M. S.; GOMES, E. C.; NASCIMENTO, F. J.; OLIVEIRA, D. A.; FONSECA, M. E.; COUTO, A. D.; SA, M. P. L.; PIRES, J. M. A. - Proteção medular em cirurgia da aorta descendente com uso de "bio-pump" e exsangüinaçāo controlada. Rev. Bras. Cir. Cardiovasc., 9 (2): 104-108, 1994.

RESUMO: Os autores utilizam como método de proteção medular em cirurgia de aorta descendente, circulação extracorpórea átrio esquerdo-femoral esquerda, com baixa dose de heparina (1 mg/kg), bomba centrifuga (bio-pump) e exsangüinaçāo controlada. Operaram 4 doentes com esta metodologia sem que houvesse mortalidade, paraplegia ou sangramento excessivo.

DESCRITORES: proteção medular em cirurgia de aorta; circulação extracorpórea; exsangüinação.

\section{INTRODUÇÃO}

A ocorrência de paraplegia após ressecção de aorta descendente para tratamento de aneurismas ou dissecção aórtica é uma complicação grave. Na enorme casuística de CRAWFORD et alii ${ }^{6}$, a ocorrência de paraplegia ou paraparesia foi de $7 \%$.

A incidência de lesão medular após cirurgia da aorta descendente está diretamente relacionada com hipotensão arterial, duração do pinçamento aórtico, aumento da pressão do líqüido céfalo-raquidiano e ligadura de artérias intercostais 1. Pinçamentos aórticos de 20-30 minutos podem provocar lesão permanente da medula. Mesmo pinçamentos aórticos de 12-15 minutos já são suficientes para provocar algum grau de disfunção medular 18 .
Vários métodos foram desenvolvidos para evitar esta complicação, como a manutenção de uma pressão arterial mínima distal ao pinçamento aórtico. Neste trabalho optamos pelo uso de circulação extracorpórea (CEC) átrio esquerdo-artéria femoral esquerda (AE-FE) sem necessidade de oxigenador 16. Substituindo a bomba de roletes clássica por uma bomba centrifuga ("bio-pump" Biomedicus) que traumatiza menos o sangue, podemos usar heparina em baixas doses ${ }^{8}$.

Os extensos trabalhos experimentais de CUNNINGHAM ${ }^{7}$ têm demonstrado a importância da pressão de perfusão medular ( $\mathrm{pPm}$ ) na etiopatogenia da paraplegia pós-operatória. A pressão de perfusão medular é dada pela diferença entre a pressão média

Trabalho realizado no Serviço de Cirurgia do Hospital Universitário Clementino Fraga Filho da Universidade Federal do Rio de Janeiro e no Serviço de Cirurgia Cardiaca do Hospital São Vicente de Paulo. Rio de Janeiro, RJ, Brasil.

Apresentado ao $21^{2}$ Congresso Nacional de Cirurgia Cardiaca. Porto Alegre, RS, 24 a 26 de março, 1994.

- Do Hospital Universitário Clementino Fraga Filho.

* Do Hospital São Vicente de Paulo.

*.. Do Hospital Universitário Clementino Fraga Filho e do Hospital São Vicente de Paulo.

Endereço para correspondência: Henrique Murad. Rua Oto de Alencar, 15, apt². 302. CEP: 20271-220 Rio de Janeiro, RJ, Brasil. 
MURAD, H.; FEITOSA, J. L.; GIAMBRONI FILHO, R.; AZEVEDO, J. A.;BRITO, J. D.; PALHARES, M. S.; GOMES, E. C.; NASCIMENTO, F. J.: OLIVEIRA, D. A.;FONSECA, M. E.; COUTO, A. D.; SA, M. P. L.; PIRES, J. M. A. - Proteção medular em cirurgia da aorta descendente com uso de "bio-pump" e exsangüinação controlada. Rev. Bras. Cir. Cardiovasc., 9 (2): 104-108, 1994.

aórtica distal ao pinçamento aórtico $(\mathrm{pAoD})$ e a pressão do líqüido cefalo-raquidiano ( $\mathrm{pLCR}$ ).

$$
\mathrm{pPm}=\mathrm{pAOD}-\mathrm{pLCR}
$$

A exsangüinação, por uma queda acentuada da $\mathrm{pLCR}$, é o método que melhor mantém a pPm, mesmo diante de uma queda da $\mathrm{PAoD}$, durante pinçamento aórtico. Cooley (ERGIN ${ }^{8}$ ) tem operado os pacientes portadores de aneurisma de aorta descendente sem uso de pinça distal, permitindo sangramento pelo coto distal.

Neste trabalho associamos as técnicas de CEC, $\mathrm{AE}-\mathrm{AF}$ com exsangüinação controlada em 4 pacientes submetidos a cirurgia de ressecção da aorta descendente. Pretendemos manter uma $\mathrm{pAoD}$ próxima do normal e a pLCR a mais baixa possível.

\section{CASUÍSTICA E MÉTODOS}

Foram operados 4 pacientes com uso de CEC, átrio esquerdo funcional e exsangüinação controlada, portadores das seguintes lesões: aneurisma de aorta descendente (1), aneurisma tóraco-abdominal (1) e dissecção aórtica (2). A idade variou de 47 a 72 anos, sendo 3 homens e 1 mulher. Dois pacientes foram operados em situação de emergência.

Foi usada intubação traqueal com tubo de duplo lume (Carlens em 2, Robertshaw em 2), para permitir colapso pulmonar esquerdo. Foi medida pressão de enchimento ventricular através de cateter de Swan-Ganz em 3 pacientes e medida direta da pressão do átrio esquerdo (por cateter colocado através da veia pulmonar esquerda) em 1 paciente. Foi medida a pressão arterial através da artéria radial direita.

Todos os pacientes foram operados em decúbito lateral direito por toracotomia póstero-lateral esquerda com ressecção da $5^{\text {a }}$ costela. No paciente com aneurisma aórtico uma $2^{\mathrm{a}}$ entrada costal foi realizada no $8^{\circ}$ espaço intercostal esquerdo, e no paciente com aneurisma tóraco-abdominal foi realizada uma toracofrenolaparotomia extraperitoneal. $O$ pericárdio foi incisado longitudinalmente, posterior ao nervo frênico esquerdo, para permitir acesso ao átrio esquerdo. A aorta foi dissecada apenas nos locais das anastomoses proximal e distal. A artéria femoral esquerda foi dissecada na região femoral esquerda.

O circuito de circulação extracorpórea (Figura 1) permite a conexão do $A E$ com a $A F$ com o uso de uma bomba centrífuga ("bio-pump" Biomedicus). Um reservatório venoso é ligado ao lado venoso da

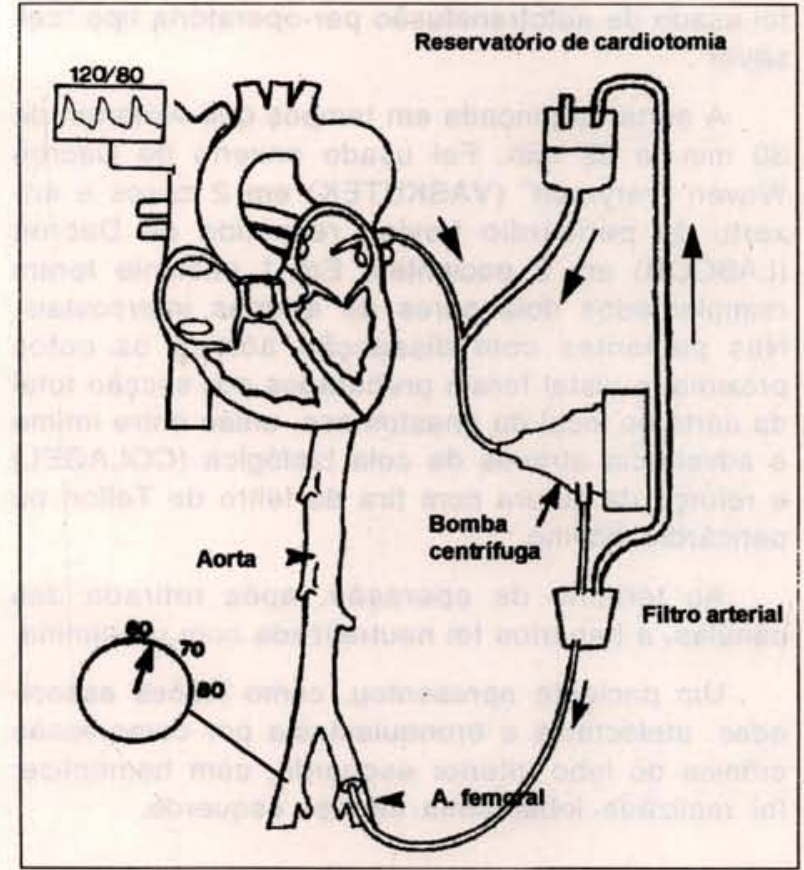

Fig. 1 - Circuito de circulação extracorpórea átrio esquerdo femoral esquerda, possibilitando exangūinação controlada para o reservatório de cardiotomia, a partir do filtro arterial.

"bio-pump" e um filtro arterial é adicionado ao lado arterial; a vasão deste filtro arterial é ligada ao reservatório de cardiotomia. Usamos $1200 \mathrm{ml}$ de solução de Ringer lactato para encher o circuito, deixando $300 \mathrm{ml}$ de solução no filtro arterial.

Usamos $1 \mathrm{ml} / \mathrm{kg}$ de heparina endovenosa, antes da introdução das cânulas. Cânula $20 \mathrm{~F}$ aramada é colocada na $A E$ através da aurícula esquerda e na $A F$, cânula apropriada a seu diâmetro. $A$ entrada em CEC se faz com infusão de líqüido para manter uma pressão arterial pulmonar média de $20 \mathrm{mmHg}$ ou uma PAE de $10 \mathrm{cmH}_{2} \mathrm{O}$. É perigosa a retirada excessiva de líqüido devido a ocorrência de hipovolemia severa. Utilizamos fluxo sangüíneo na bomba de $1500 \mathrm{ml} / \mathrm{min}$. A aorta descendente é pinçada após estabilização da CEC.

O controle da hipertensão arterial proximal ao pinçamento aórtico foi feito através de exsangüinação controlada pela vasão do filtro arterial. Retiradas de $100 \mathrm{ml}$ a $300 \mathrm{ml}$ de sangue eram por vezes suficientes para manter a pressão sistólica proximal ao redor de $100 \mathrm{mmHg}$ e episódios de hipotensão arterial proximal eram tratados com transfusão de sangue a partir do reservatório de cardiotomia. Não foi usado nitroprussiato de sódio. $O$ sangue coletado no campo operatório era aspirado para o reservatório de cardiotomia. Em 1 paciente 
MURAD, H.; FEITOSA, J. L.; GIAMBRONI FILHO, R.; AZEVEDO, J. A.;BRITO, J. D.; PALHARES, M. S.; GOMES, E. C.; NASCIMENTO, F. J.; OLIVEIRA, D. A.;FONSECA, M. E.; COUTO, A. D.; SA. M.P. L.;PIRES, J. M. A. - Proteção medular em cirurgia da aorta descendente com uso de "bio-pump" e exsangüinaçăo controlada. Rev. Bras. Cir. Cardiovasc., 9 (2): 104-108, 1994.

foi usado de autotransfusão per-operatória tipo "cell saver".

A aorta foi pinçada em tempos que variaram de $30 \mathrm{~min}$. a $62 \mathrm{~min}$. Foi usado enxerto de Dacron Woven "very-soft" (VASKUTEK) em 2 casos e enxerto de pericárdio bovino revestido de Dacron (LABCOR) em 2 pacientes. Em 1 paciente foram reimplantados dois pares de artérias intercostais. Nos pacientes com dissecção aórtica os cotos proximal e distal foram preparados por secção total da aorta no local da anastomose, uniāo entre intima e adventícia através de cola biológica (COLAGEL) e reforço da sutura com tira de feltro de Teflon ou pericárdio bovino.

Ao término da operação, após retirada das cânulas, a heparina foi neutralizada com protamina.

Um paciente apresentou, como lesões associadas, atelectasia e bronquiectasia por compressão crônica do lobo inferior esquerdo, com hemoptise; foi realizada lobectomia inferior esquerda.

\section{RESULTADOS}

Não houve mortalidade, paraplegia ou paraparesia neste grupo de pacientes. O sangramento per-operatório não foi significativo em nenhum paciente, sendo que em um deles não houve necessidade de transfusão sangüinea. A drenagem torácica pós-operatória variou de $300 \mathrm{ml}$ a $700 \mathrm{ml}$.

No paciente em que foi feita lobectomia inferior esquerda houve atelectasia pulmonar esquerda, necessitando broncoscopia e, posteriormente, toracoscopia para evacuação de coágulos pleurais.

\section{COMENTÁRIOS}

Diversos trabalhos têm demonstrado uma relação direta entre o tempo de pinçamento da aorta descendente e a incidência de paraplegia. Tempos de pinçamento superiores a 30 minutos são acompanhados por uma incidência maior de paraplegia 13 .

A CEC átrio esquerdo-femoral permite manutenção de pressão arterial superior a $40 \mathrm{mmHg}$ distal ao pinçamento aórtico, com a vantagem de não necessitar de oxigenador. Com o uso de bomba centrifuga realizamos a operação com doses baixas de heparina e o sangramento, que é uma complicação importante neste tipo de pacientes, pôde, deste modo, ser evitado.

Com o sistema usado neste trabalho, precozinado por COSELLI ${ }^{5}$ e ERGIN ${ }^{8}$, conseguimos um controle bastante adequado da pré-carga, da pós- carga e da perfusão aórtica distal ao pinçamento aórtico sem importante manipulação medicamentosa.

Algumas das várias técnicas adicionais recomendadas para proteção medular foram usadas nestes pacientes: a) reimplante de artérias intercostais entre T8 e L2, particularmente importantes e com pouco sangramento de retorno 10 ; b) metilprednisolona $30 \mathrm{mg} / \mathrm{kg}$ EV antes do pinçamento aórtico ${ }^{11}$; c) evitar hipo ou hipertensão arterial ${ }^{17}$.

O pinçamento aórtico produz hipertensão arterial proximal, aumento da pressão do líqüido cefaloraquidiano (LCR) e a hipotensão arterial distal. $\mathrm{O}$ aumento súbito da pós-carga tem conseqüências deletérias sobre o coração com isquemia miocárdica e falência ventricular.

$A$ pressão de perfusão medular $(\mathrm{pPm}=\mathrm{pAoD}$ = pLCR é importante, pois, para uma perfusão medular adequada, devemos manter a $\mathrm{PAOD}$ próxima do normal e uma pLCR a mais baixa possivel. A manutenção de $\mathrm{pAOD}$ próxima do normal pode ser obtida com a CEC átrio esquerdo-femoral.

A pressão normal do LCR varia de $5 \mathrm{mmHg}$ a $10 \mathrm{mmHg}$; ele é produzido nos plexos coróides na velocidade de $20 \mathrm{ml} / \mathrm{h}$ e a sua drenagem é feita pelas vilosidades aracnóides para o sistema venoso. Em experimentação animal, a pLCR aumenta com o pinçamento da aorta descendente. O nitroprussiato de sódio e a nitroglicerina usados para controlar a hipertensão arterial proximal ao pinçamento aórtico alteram a auto-regulação cerebral aumentando o fluxo sangüíneo cerebral e a pressão do LCR ${ }^{14}$. CERNAIANU et alii ${ }^{3}$ demonstraram em cães o efeito deletério do nitroprussiato de sódio sobre a perfusão medular, pois diminui ainda mais o fluxo sangüineo medular proveniente de colaterais, além de permitir a abertura de fístulas artério-venosas, diminuindo a perfusão capilar.

Os trabalhos de CONNOLLY ${ }^{4}$ e de CUNNINGHAM ${ }^{7}$ demonstraram que a exsangüinação controlada diminui a pLCR e aumenta a pPm mesmo em condiçōes de $p A o D$ baixa. Outra técnica usada para diminuir o pLCR é a drenagem do LCR. Em nossos pacientes achamos a técnica de exsangüinação controlada mais simples de ser executada.

A lesão que ocorre após isquemia medular é agravada pela reperfusão. Ocorre uma síndrome compartimentar com edema, aumento da pLCR e agravamento da lesão celular. Este fato torna ainda mais importante nossos esforços para diminuir o pLCR.

A operação clássica para o tratamento de lesão de aorta descendente apresentava alta mortalidade (35\%) em pacientes com dissecção de aorta tipo B de Stanford (GLOWER et alii ${ }^{9}$ ). Isto propiciou o aparecimento de operações alternativas: trombo- 
MURAD, H.; FEITOSA, J. L.; GIAMBRONI FILHO, R.; AZEVEDO, J. A.; BRITO, J. D.; PALHARES, M. S.; GOMES, E. C.; NASCIMENTO, F. J.;OLIVEIRA, D. A.;FONSECA, M. E.; COUTO, A. D.; SA. M. P. L.; PIRES. J. M. A. - Proteção medular em cirurgia da aorta descendente com uso de "bio-pump" e exsangüinação controlada. Rev. Bras. Cir. Cardiovasc., 9 (2): 104-108, 1994.

exclusão, proposta por CARPENTIER et alii ${ }^{2}$ e tromba de elefante, proposta por PALMA et alii ${ }^{15}$, com mortalidades em torno de $25 \%$.

Entretanto, SVENSON et alii ${ }^{18}$, usando a cirurgia clássica em dissecção aguda de aorta tipo B de Stanford, relataram mortalidade de apenas $6 \%$ para pacientes operados entre 1984 a 1989 . O grupo de Stanford (MILLER ${ }^{12}$ ) em pacientes selecionados (sem complicações pré-operatórias de dificil controle, como isquemia mesentérica), obteve mortalidade de $5 \%$.

Torna-se importante um método facilmente reprodutível que permita resultados excelentes. $O$ uso de CEC, AE-AF com exsangüinação controlada parece ser a solução para esta necessidade, como mostram os nossos resultados.

Outra preocupação do cirurgião é a evolução tardia. Com a operação clássica há uma incidência de $13 \%$ de mortalidade tardia nos pacientes com dissecção aguda por rotura da aorta ou de falso aneurisma ${ }^{12}$. Interroga-se o futuro dos pacientes submetidos a colocação de tromba de elefante durante dissecções agudas.

\section{CONCLUSÕES}

- O uso de CEC, AE-AF permite a obtenção de pressão aórtica distal adequada sem necessidade do uso de oxigenador.

- O uso de bomba centrifuga ("bio-pump" Biomedicus) favorece as baixas doses de heparina, o que contribui para um menor sangramento per-operatório.

- Com o uso de exsangüinação controlada é possivel controlar a pós-carga e manter uma pressão de perfusão medular suficiente para se evitar lesōes neurológicas.

- Não houve mortalidade ou presença de lesões neurológicas nos pacientes operados.

MURAD, H.; FEITOSA, J. L.; GIAMBRONI FILHO, R.; AZEVEDO, J. A.; BRITO, J. D.; PALHARES, M. S.; GOMES, E. C.; NASCIMENTO, F. J.; OLIVEIRA, D. A.; FONSECA, M. E.; COUTO, A. D.; SÁ, M. P. L.; PIRES, J. M. A. - Spinal protection during descending aortic surgery. Rev. Bras. Cir. Cardiovasc., 9 (2): 104-108, 1994.

ABSTRACT: The authors have used for spinal cord protection during descending aortic surgery, partial extracorporeal circulation from left atrium to left femoral artery, low dose of heparin $(1 \mathrm{mg} / \mathrm{kg})$, centrifugal pump (bio-pump) and controlled exsanguination. They have operated upon with this methodology 4 patients without mortality, paraplegia or excessive bleeding.

DESCRIPTORS: spinal protection in aortic surgery; extracorporeal circulation; exsanguination.

\section{REFERÊNCIAS BIBLIOGRÁFICAS}

1 BERKOFF, H. A. \& FOLLETTE, D. A. - Paraplegia associated with thoracic aortic surgery. Chest Surg. Clin. N. Am., 2: 379-381, 1992.

CARPENTIER, A.; DELOCHE, A.; FABIANI, J. N.; et al.: New surgical approach to aortic dissection: flow reversal and tromboexclusion. J. Thorac. Cardiovasc. Surg., 81: 659-668, 1981. Effect of sodium nitroprusside on paraplegia during cross-clamping of the thoracic aorta. Ann. Thorac. Surg., 56: 1035-1037, 1993.

(1)

A prospective randomized study of cerebrospinal fluid
drainage to prevent paraplegia after high-risk surgery
on the thoracoabdominal aorta. J. Vasc. Surg., 13: $36-45,1991$.

7 CUNNINGHAM, J. N. - Spinal cord protection during 
MURAD, H.; FEITOSA, J. L.; GIAMBRONI FILHO, R.; AZEVEDO, J. A.; BRITO, J. D.; PALHARES, M. S.; GOMES, E. C.; NASCIMENTO, F. J.; OLIVEIRA, D. A.; FONSECA, M. E.; COUTO, A. D.; SA, M.P. L.; PIRES, J. M. A. - Proteção medular em cirurgia da aorta descendente com uso de "bio-pump" e exsangüinação controlada. Rev. Bras. Cir. Cardiovasc., 9 (2): 104-108, 1994.

thoracic aortic surgery. Aortic Surgery. Symposium, 3. New York, abril 1992.

8 ERGIN, M. A. - Descending aneurysm: methods of protection. Aortic Surgery Symposium, 3. New York, april, 1992.

9 GLOWER, D. D.; SPEIER, R. F.; WHITE, W. D.; et al.: Management and long term outcome of aortic dissection. Ann. Surg., 214: 31-41, 1991.

10 KIEFFER, E.; RICHARD, T.; CHICAS, J.; et al.: Preoperative spinal cord arteriography in aneurysmal disease of the descending thoracic and thoracoabdominal aorta: preliminary results in 45 patients. Ann. Vasc. Surg., 3: 34-39, 1989.

11 LASCHINGER, J. C.; CUNNINGHAM Jr., J. N.; COOPER, M. M.; et al.: Prevention of ischemic spinal cord injury following aortic cross-clamping. Use Of corticosteroids. Ann. Thorac. Surg., 38: 500-507, 1984.

MILLER, D. C. - The continuing dilema concerning medical versus surgical management of patients with acute type 3 dissections. Sem. Thorac. Cardiovasc. Surg., 5: 33-46, 1993.

13 MURAD, H. - Estudo dos métodos para prevenir isquemia medular em cirurgia da aorta descendente. Rio de
Janeiro, RJ, 1992. [Tese. Mestrado. Universidade Federal do Rio de Janeiro].

14 MYHRE, H. O.; SAETHER, O. D.; STENSETH, R. Attempts to reduce paraplegia risk. In: Aortic surgery in the cause and management of aneurysms. GREENHALGH, R. M. \& MANICK, J. A. (eds.) W. B. Saunders Co., London, 1990. p. 373-385.

PALMA, H.; JULIANO, J. A.; CAL, R. G. R.; ALMEIDA, D. R.; OTA, L. H.; GIANOTTI, I. A.; ANDADE, J. C. S.; BUFFOLO, E. - Tratamento dos aneurismas da aorta descendente por endoprótese (tromba de elefante). Rev. Bras. Cir. Cardiovasc., 4: 190-194, 1989.

16 SVENSON, L. G. \& CRAWFORD, E. S. - Aortic dissection and aortic aneurysm surgery: clinical observations, experimental investigations and statistical analysis. Part I. Curr. Probl. Surg., 29 (11): 817-911, 1992.

17 SVENSON, L. G. \& CRAWFORD, E. S. - Aortic dissection and aortic aneurysm surgery: clinical observations, experimental investigations and statistical analysis. Part III. Curr. Probl. Surg., 30 (1): 1-163, 1993.

18 SVENSON, L. G.; CRAWFORD, E. S.; HESS, K. R.; et al.: Dissection of the aorta and dissecting aneurysms: improving early and long-term surgical results. Circulation, 82 (Supl. 4): 24-38, 1990. 